\title{
Assessment of physician-assisted death by members of the public prosecution in the Netherlands
}

Jacqueline M Cuperus-Bosma, Gerrit van der Wal, Caspar W N Looman, and Paul J van der Maas Vrije Universiteit Amsterdam, Amsterdam, the Netherlands and Erasmus University, Rotterdam, the Netherlands

\author{
Abstract \\ Objectives-To identify the factors that influence the \\ assessment of reported cases of physician-assisted \\ death by members of the public prosecution. \\ Design/setting - At the beginning of 1996, during \\ verbal interviews, 12 short case-descriptions were \\ presented to a representative group of 47 members of \\ the public prosecution in the Netherlands. \\ Results-Assessment varied considerably between \\ respondents. Some respondents made more "lenient" \\ assessments than others. Characteristics of the \\ respondents, such as function, personal-life philosophy \\ and age, were not related to the assessment. Case \\ characteristics, ie the presence of an explicit request, \\ life expectancy and the type of suffering, strongly \\ influenced the assessment. Of these characteristics, the \\ presence or absence of an explicit request was the most \\ important determinant of the decision whether or not \\ to hold an inquest. \\ Conclusions-Although the presence of an explicit \\ request, life expectancy and the type of suffering each \\ influenced the assessment, each individual assessment \\ was dependent on the assessor. The resulting danger \\ of legal inequality and legal uncertainty, particularly \\ in complicated cases, should be kept to a minimum by \\ the introduction of some form of protocol and \\ consultation in doubtful or boundary cases. The \\ notification procedure already promotes a certain \\ degree of uniformity in the prosecution policy. \\ (Fournal of Medical Ethics 1999;25:8-15)
}

Keywords: Assessment; euthanasia; notification proce-dure; physician-assisted death; public prosecutor; vignettesio

\section{Introduction}

In the Netherlands, physician-assisted death (fig T⿱乛龰 ure 1) is still subject to criminal law. In the past? two decades, however, criteria for accepted⿳亠丷厂 practice have been formulated by the Dutch courts and the medical profession. In generalse there will be no prosecution if these criteria have been met: the patient must experience his or hero suffering as unbearable and hopeless; the request must be well considered, persistent and voluntary;ō the physician must consult at least one other phy sician, and the physician is not allowed to issue a certificate testifying to natural death, and isôㄹ obliged to keep records.

Since 1991 (officially since June 1994) a notifi cation procedure for physician-assisted death has been in use (figure 2). The most important objective of this procedure is to encourage physicians too disclose their practice of assisting with death. In addition, it is the intention that uniformity will beo achieved in notification and that reported cases of physician-assisted death will be dealt with uni-음 formly throughout the country. Up until 1991, if cases were reported, they were reported in various ways, either to the police, the coroner or the pub-N lic prosecutor.

The notification procedure requires doctors to report each case of physician-assisted death to the

Figure 1 Definitions of physician-assisted death, ie euthanasia, physician-assisted suicide and ending of life without an explicit request

\section{Euthanasia}

Physician-assisted suicide

Ending of life without an explicit request
Administration of drugs with the explicit intention of ending the patient's life, at the patient's explicit request

The prescription or supplying of drugs with the explicit intention of enabling the patient to end his or her own life

Administration of drugs with the explicit intention of ending the patient's life without a concurrent, explicit request by the patient

We do not consider the following activities to be physician-assisted death:

- withholding or withdrawing life-prolonging treatment at the explicit and earnest request of the patient,

- withholding or withdrawing life-prolonging treatment that is medically futile,

- performing a medical act or administering a drug intended to alleviate unbearable suffering (for example a painkiller), even if one of

the side effects may be hastening death. \section{(1)}

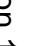




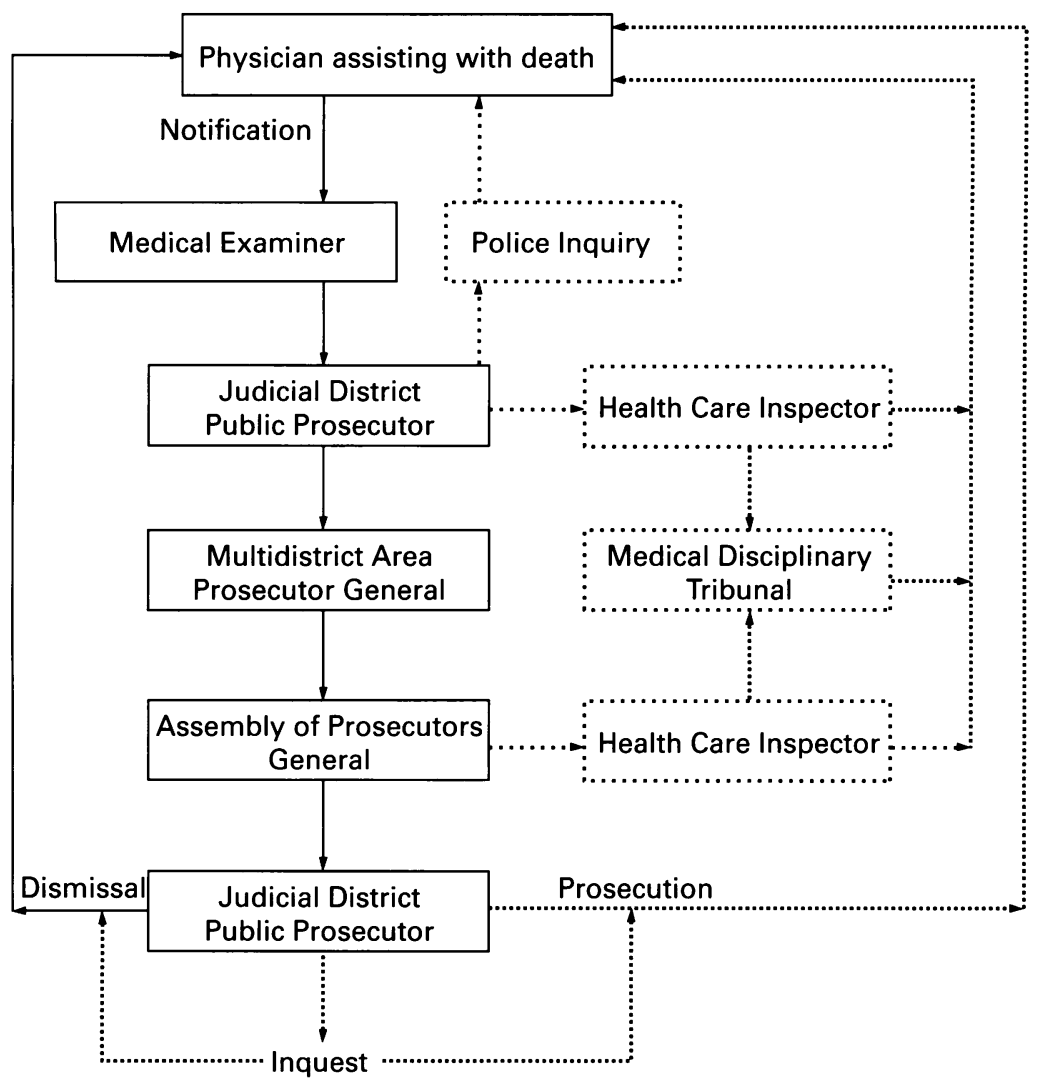

Figure 2

Common procedure

Procedure on indication

coroner who, in turn, informs the public prosecutor of the judicial district in which it took place (of which there are 19 in he Netherlands). The public prosecutor presents his assessment to the prosecutor general, the head of the multidistrict area (there are five such multidistricts in the Netherlands, each consisting of three or four judicial districts). The prosecutor general, usually assisted by an advocate general, presents the case to the assembly of prosecutors general (consisting of the five prosecutors general). The assembly makes a preliminary decision whether to prosecute, and whether to involve a health inspector, for example, for advice or in order to call a disciplinary hearing. The minister of justice is responsible for the final decision. Although the uniformity in notification to the coroner has increased due to the establishment of the notification procedure, and the number of reported cases of euthanasia and physician-assisted suicide has increased from $18 \%$ in 1990 to $41 \%$ in $1995,60 \%$ of the cases are still not reported. Notification of cases without an explicit request for euthanasia having been made by the patient remained very low. The main reasons given by doctors for not reporting a case of physician-assisted death were to do with their reluctance to become involved in legal procedures. $^{2}$

Elsewhere we have described the considerations of the assembly of prosecutors general in dealing with cases reported during the period 1991-1995. In this article we will make use of specially designed case histories to determine the extent to which the assessment of physicianassisted death varies between the various (categories of) respondents of the public prosecution and between cases, and whether characteristics of respondents and of cases can explain this variation.

\section{Methods}

STUDY POPULATION

Within the framework of the nationwide evaluation study of the euthanasia notification procedure that was commissioned by the minister of 
Figure 3 Variables in the 12 vignettes

\begin{tabular}{|c|c|c|c|c|c|c|c|c|c|c|c|c|}
\hline Vignette number & 1 & 2 & 3 & 4 & 5 & 6 & 7 & 8 & 9 & 10 & 11 & 12 \\
\hline Suffering & Pain & $\begin{array}{l}\text { Loss of } \\
\text { dignity }\end{array}$ & $\begin{array}{l}\text { Loss of } \\
\text { dignity }\end{array}$ & Pain & Pain & $\begin{array}{l}\text { Loss of } \\
\text { dignity }\end{array}$ & $\begin{array}{l}\text { Loss of } \\
\text { dignity }\end{array}$ & Pain & Pain & $\begin{array}{l}\text { Loss of } \\
\text { dignity }\end{array}$ & $\begin{array}{l}\text { Loss of } \\
\text { dignity }\end{array}$ & Pain \\
\hline Life expectancy & Medium & Medium & Long & Long & Short & Long & Short & Medium & Short & Medium & Short & Mediun \\
\hline Explicit request & & Yes & Yes & Yes & ? & $?$ & ? & ? & No & No & No & No \\
\hline
\end{tabular}

public health, welfare and sport and the minister of justice, 48 members of the public prosecution were interviewed at the beginning of 1996 by specially trained lawyers. Adequate representation from each of the 19 judicial districts was obtained by interviewing two public prosecutors from each judicial district and three public prosecutors from three large judicial districts. Eventually a total of 39 public prosecutors were interviewed: two public prosecutors could not participate, one because of prolonged illness and one because of personal circumstances. One respondent did not complete this part of the interview because of time restrictions. In four of the five multidistrict areas the reported cases were mainly dealt with by one advocate general; in one multidistrict area they were dealt with by the prosecutor general himself. The four advocates general and the five prosecutors general were all interviewed.

\section{MEASURING INSTRUMENT}

During the interviews these 47 respondents were presented with 12 short case histories (vignettes) in which physician-assisted death took place. Each vignette was read out by the interviewer, after which the respondent was asked: "If the physician reported this case to the coroner, what would be your recommendation for further action?" (see appendix). By presenting the same vignettes to all respondents, we were able to receive a more general impression of the way in which members of the public prosecution assess cases of physicianassisted death. Also we were able to investigate the assessment of "boundary-breaking" situations.

In the introduction to this section of the questionnaire the respondents were asked to imagine the situation described to them as clearly as possible. The interviewer explained that for reasons of comparability no further information about the case could be provided than the description given in the vignette. The respondent had to assume that symptoms and alternative methods of treatment which had not been mentioned did not exist, and that the requirements for careful practice which had not been mentioned had been met (including consultation of another physician and prudent performance).

In the vignettes there was variation with regard to type of suffering (pain or loss of dignity); life expectancy (short: $\leqslant 1$ week; medium: $\leqslant 1$ month; long: $\geqslant 6$ months), and the patient $\stackrel{\text { s }}{s}$ request (explicit request, unclear request or no request).

The variable type of suffering was chosen to investigate to what extent certain aspects of the patient's suffering play a role in the assessment of the question whether there is "unbearable and" hopeless suffering" (one of the requirements foio careful practice). Furthermore we were interesteds in determining to what extent life expectancy plays a role in the assessment of cases. Althought the Supreme Court determined in 1994 that the presence of a terminal phase is not a necessare requirement for unpenalised physician-assisted death, there had been a lot of discussion on thise subject in the Netherlands up to that time. In fact; the minister of justice at that time was of the opin-0 ion that the presence of a terminal phase was as necessary requirement for careful practice and wanted to have the physician prosecuted when this criterion had not been met. The variable patient's request was chosen to see to what exten $\vec{\rho}$ the presence or absence of an explicit request (one of the requirements for careful practice) plays af role in the assessment.

With these three variables a total of 18 differen combinations (vignettes) are possible. Of these, 120 have been presented. Because the vignettes were $\mathrm{a}$ part of a much longer interview, six vignetteso could not be presented because of time restric 3 tions. The answers to these remaining vignettes? were statistically estimated.

\section{ANALYSIS}

The choice of answers presented on a card consisted of seven categories:

1. dismissal - no prosecution or other action required;

2. dismissal, but a disciplinary hearing by the health inspector ordered;

3. advice from the health inspector requested;

4. inquest ordered;

5. advice from experts requested;

6. summons issued, and

7. other, namely:

In the data-analysis the category "other, namely ..." is sub-divided into two categories:

7.1. advice from the coroner, physician, partner of the deceased and/or other persons re을 quested, and 
Table 1 The assessment of 12 vignettes by 47 members of the public prosecution

\begin{tabular}{|c|c|c|c|c|c|c|c|c|c|c|c|c|}
\hline Vignette number: & 1 & 2 & 3 & 4 & 5 & 6 & 7 & 8 & 9 & 10 & 11 & 12 \\
\hline Dismissal & 47 & 39 & 27 & 37 & 19 & 3 & 9 & 31 & - & 1 & 5 & 3 \\
\hline $\begin{array}{l}\text { Dismissal, hearing by Health Inspector ordered } \\
\text { Advice from Coroner, physician, partner }\end{array}$ & - & 1 & - & 1 & 1 & 1 & 1 & - & 3 & 3 & 3 & 2 \\
\hline requested ${ }^{\star}$ & - & 2 & 5 & 3 & 13 & 8 & 7 & 5 & 4 & 2 & 2 & 3 \\
\hline Advice from Health Inspector requested & - & 5 & 8 & 6 & 3 & 11 & 8 & 4 & 2 & 1 & 5 & 3 \\
\hline Order police inquiry & - & - & - & - & - & - & 1 & 3 & 3 & 3 & 2 & 4 \\
\hline Inquest ordered & - & - & - & - & 9 & 15 & 16 & 4 & 19 & 21 & 17 & 20 \\
\hline Advice from experts requested & - & - & 7 & - & 2 & 5 & 2 & - & - & - & - & 1 \\
\hline Summons issued & - & - & - & - & - & 4 & 3 & - & 16 & 16 & 13 & 11 \\
\hline
\end{tabular}

*The majority of advocates general and prosecutors general indicated that the request for advice would be arranged by the public prosecutor.

\section{2 order police inquiry.}

To perform logistic regression analysis we reduced the eight decision options to two groups:

1. Recommendation for a dismissal or a request for advice:

dismissal, dismissal but a disciplinary hearing by the health inspector ordered, advice from the health inspector requested and the "other, namely

..." in which the coroner, the physician, the partner of the deceased and/or other persons are asked for advice.

2. Recommendation for an inquest:

inquest ordered, advice from experts requested, summons issued and police inquiry ordered.

The reason for this sub-division was that in category 1 the case is not (yet) considered to be (or likely to be) subject to prosecution. In category 2 the physician will be prosecuted, or there is a reasonable chance that prosecution will follow.

Although the observations were not independent of each other, this has not been taken into account in the calculations. We applied Generalised Estimated Equations to investigate whether this made any difference, but the effect of the dependence appeared to be small: the outcome remained almost the same. ${ }^{4}$

In order to determine whether certain respondents made a more "lenient" assessment than others, we evaluated the eight answer categories on a scale of "leniency" ranging from 1 to 10 : (most "lenient") 1: dismissal, 2: dismissal, but a disciplinary hearing by the health inspector requested, 3 : advice from the coroner, physician, partner and/or other persons requested (excluding the health inspector or experts), 4: advice from the health inspector requested, 7: order police inquiry, 8: advice from experts requested or an inquest ordered, and (least "lenient") 10: summons issued. Obviously this sequence and attribution of values is open to debate, but seemed to us a reasonable way of grading the legal assessors' "leniency". We adhered as much as possible to the sequence which was presented to the respondents on the card because this sequence could be suggestive of an order of importance. Since, in fact, expert advice is always requested at an inquest, these two categories were attributed the same value. For each respondent the average score for the 12 vignettes was calculated.

\section{RESULTS}

Are there differences in the assessments of the various respondents of the public prosecution?

Table 1 shows that there was considerable variation in the answers given. The largest variation was found among the public prosecutors. It is remarkable that there were vignettes to which the answer of some respondents was "dismissal", whereas according to other respondents an inquest should be held or the physician should be summoned immediately. This was the case, for instance, with vignette 11 (loss of dignity, a maximum life expectancy of one week and no request from the patient, see also vignette 7 ). There was only complete agreement about the first vignette, where all respondents opted for "dismissal". "Order police inquiry" was only opted for by public prosecutors. This was also the case, with one exception, for "dismissal, but a disciplinary hearing by the health inspector ordered" and to "advice from experts requested". "Summons issued" was only given as an answer if there was no request from the patient or if the request was unclear and accompanied by suffering in the form of loss of dignity.

An average score per vignette was calculated for each respondent (minimum 1 and maximum 10), in order to determine whether certain respondents gave a more "lenient" assessment than others. For both the 37 public prosecutors (average score ranging from 1.4 to 6.9 ) and the nine advocates general and prosecutors general (average score ranging from 1.8 to 6.3 ) this proved to be the case. For instance, two respondents recommended dismissal in nine of the cases, while two other respondents recommended a summons in six cases. 
Are there differences in assessment between the public prosecutors and the advocates general and prosecutors general?

In order to determine whether there was a difference in assessment between the public prosecutors (judicial districts), on the one hand, and the advocates general and the prosecutors general (multidistrict areas), on the other hand, logistic regression analysis was performed. ' For both groups of respondents the probability of the assessment "recommendation for an inquest" was calculated for the total of 12 vignettes (ie 564 observations). Information about advocates general and prosecutors general was combined because of the small number of respondents in each group. For the public prosecutors the "overall" chance that they would recommend an inquest was 39\% (179/456) and for the advocates general and the prosecutors general it was $35 \%$ $(38 / 108)$. The difference was not statistically significant $(\mathrm{p}$-value $=0.43)$.

The relation between characteristics of public prosecutors and their assessment of physician-assisted death

In a similar way we investigated whether there was a difference between the assessment of the public prosecutors who hold "final responsibility" in the judicial districts (ie those who send the judgment to the multidistrict courts, $n=16$ ) and the picket officers (ie those who receive the reports from the coroner, $n=22$ ). In both groups the chance of a "recommendation for an inquest" was 39\%.

There was also no difference found between the 20 public prosecutors who considered themselves to belong to a religious group or said they had a certain personal philosophy of life, such as Roman Catholic or Protestant, and the other 18 respondents.

Finally, we investigated whether the age of the respondents was related to their assessment by dividing them into "under 50 " $(\mathrm{n}=20)$ and 50 years and older $(n=18)$ categories. There was no difference in assessment between these groups.

\section{The relation between vignette characteristics and the} assessment

By means of logistic regression analysis, we studied the relation between the three vignette variables (explicit request, life expectancy and type of suffering) and the chance of a "recommendation for an inquest". All univariate associations were statistically significant. Subsequently, we estimated the combined effects of the three variables (multivariate regression analysis). All variables then appeared to have a significant association with the "recommendation for an
Table 2 Chance of a 'recommendation for an inquest' according to the variables of suffering, life expectancy and explicit request (percentages)

\begin{tabular}{|c|c|c|c|c|c|c|}
\hline \multirow{2}{*}{$\frac{\text { Suffering }}{\text { Life expectancy }}$} & \multicolumn{3}{|l|}{ Pain } & \multicolumn{3}{|c|}{ Los of dignity } \\
\hline & Short & Medium & Long & Short & Medium & \\
\hline $\begin{array}{l}\text { Explicit request } \\
\text { - present }\end{array}$ & 2 & 2 & $t$ & 3 & 3 & $\bar{T}$ \\
\hline - unclear & $2 \overline{4}$ & $2 \overline{1}$ & 41 & 36 & 32 & 55 \\
\hline - absent & 75 & 71 & 87 & 84 & 81 & 92 \\
\hline
\end{tabular}

inquest". The association was most pronounced with the explicit request being present or absenti$(p$-value $=0.00)$, followed by the type of suffering $(\mathrm{p}$-value $=0.02)$ and the life expectancy ( $\mathrm{p}_{-}$ value $=0.04)$. Further analysis showed that for life expectancy only the difference between "long" $\left(\geqslant \frac{\mathbb{O}}{2}\right.$ six months) versus "medium" ( $\leqslant$ one month) anct "short" ( $\leqslant$ one week) was significantly associated."

Table 2 presents the probability of a "rec- $\vec{\emptyset}$ ommendation for an inquest" for all 18 combina 8 tions which can be made with the three variables. The calculations are based on the assumption that the effects of the three variables are independento From table 2 it is clear that the chance of a "rec $\frac{0}{\nabla}$ ommendation for an inquest" was lowest wher $\mathbb{Q}$ there was an explicit request $(2-7 \%)$. The chance $\overrightarrow{\overrightarrow{0}}$ of a "recommendation for an inquest" was greater if the request was unclear $(21-55 \%)$, and in creased to $71-92 \%$ if there had been no reques from the patient. A life expectancy of six months or longer implies a greater chance of a "rec-0 ommendation for an inquest" than a "short" or. "medium" life expectancy. With regard to the typeo of suffering, loss of dignity implied a greater chance of a "recommendation for an inquest" than pain which could not be alleviated.

Finally, we investigated the possible interaction. between variables. In order to perform theseos analyses the life expectancies "short" and "meN dium" were combined. Only the interaction N between the type of suffering and the presence or absence of a request was significant - just 6 $(p$-value $=0.05)$. In case of long life expectancy the type of suffering seemed to play a more important? role if the request was unclear: with pain the chance of a "recommendation for an inquest" was. smaller than with loss of dignity $(30 \%$ versus $56 \%$ ). This also applied in the presence of an explicit request $(0 \%$ versus $9 \%)$. On the other hand, the type of suffering played no role in theo absence of an explicit request $(87 \%$ versus $86 \%)$ This pattern was the same with a life expectancto of less than one month. 


\section{Discussion}

These results give an interesting insight into how members of the public prosecution examine cases of physician-assisted death. All the judicial districts were adequately represented. Moreover, all advocates general, who are responsible for the examination of reported cases, and all prosecutors general, were interviewed. All vignettes presented to the respondents described situations in accordance with real life. However, in real life not all combinations of variables occur as often. Vignettes 1 and 2 represented the majority of reported cases. The other vignettes concerned the more complicated cases on the boundaries of medical practice of which the assessment is not clear at this moment and which are (probably) not reported yet. We used the methodology of vignettes to obtain general insight into the uniformity of assessment of both simple and more complicated cases by members of the public prosecution. Although the respondents often indicated their wish to have more details of the case before being able to judge them, the following conclusions seem grounded.

The type of suffering, the presence of an explicit request and the expected life expectancy of the patient all play a role in the way in which the members of the public prosecution assess physician-assisted death. Of these three aspects, the presence or absence of an explicit request from the patient is the most conclusive factor in the decision whether or not to recommend an inquest. This is in line with the fact that the presence of a voluntary and well considered request from the patient is a central element in jurisprudence with regard to the assessment of physicianassisted death. Moreover, it reflects the enormous importance attached to the principle of autonomy in the ethical and legal debate about physicianassisted death. ${ }^{6}$ Also in view of the statement by the previous minister of justice, that physicians reporting cases that did not involve the explicit request of the patient would always be prosecuted, this is not surprising.

Our study shows that a life expectancy of six months or more implies a greater chance of a "recommendation for an inquest" than a life expectancy of one month or less. This is remarkable, since the Chabot verdict by the Supreme Court (1994) states that the terminal phase is not a necessary criterion for unpenalised euthanasia. ${ }^{7}$ Thus, although in strictly legal terms it is not correct that the expected life expectancy of the patient should play such an important role in the assessment of the cases, apparently a lot of members of the public prosecution appear to continue to place much importance on this aspect. It might be that personal moral or psychological reasons play a role in this.

With regard to the type of suffering, the "loss of dignity" aspect results in a greater chance of a "recommendation for an inquest" than in the case of "pain" which cannot be alleviated. According to the requirements for careful practice there has to be unbearable and hopeless suffering of the patient. This criterion has not yet been clearly defined. Apparently, the respondents felt more certain about pain which could not be alleviated, being unbearable and hopeless than about loss of dignity, which caused unbearable and hopeless suffering. This has major implications for doctors who respond in good faith to a "loss of dignity" request for euthanasia, since their chances of an inquest or other actions are considerably greater.

Although case characteristics played an important role in the assessment of cases, the divergence in assessment of most of the cases between respondents was considerable. It is important to note that some respondents indicated, for most vignettes, that they would recommend dismissal, whereas others would more often issue a summons or hold an inquest. At group level, however, there appeared to be no (significant) differences in assessment between public prosecutors, on the one hand, and advocates and prosecutors general, on the other hand, nor between public prosecutors who bear the final responsibility of assessment at the judicial districts and the "initial assessment" officers. The personal philosophy of life of the public prosecutors and their age were also not related to the assessment. Therefore the heterogeneity has to depend on other characteristics of the respondents. In terms of uniformity of examination, this variation implies the risk of legal inequality and of legal uncertainty. In more complicated cases members of the public prosecution might assess the same case differently. However, the vast majority of cases presented to the public prosecution are less complicated than those described in most of the vignettes.

Moreover, the method by which all reported cases are eventually dealt with by the assembly of prosecutors general promotes a certain amount of uniformity in the prosecution policy, which prevents legal inequality to a large extent. The disadvantage of this stepwise examination is that it increases the period between notification by the physician and the moment when the physician is informed about the decision whether the case will be dismissed. This period lasts for an average of 103 days for cases which are dismissed directly by the assembly. In cases for which an inquest is held, or in which the physician is summoned, it takes much longer, sometimes even years. ${ }^{2}$ 
The uniformity of examination by the members of the public prosecution would be enhanced by the development of a protocol. Explicit specification of the criteria which form the basis of judicial prosecution will help to increase legal security and legal equality in more complicated cases.

The government has developed a new notification procedure. In this procedure initial examination will be done by regional multidisciplinary assessment committees (which will include a physician, a lawyer and an ethicist). These committees will make important recommendations concerning the outcome of each reported case to the public prosecution. This implies that the role of the public prosecution will decrease. Although there will be a change in the way in which physician-assisted death is examined, our study illustrates the necessity of open and explicit standards for the legal examination of reported cases of physician-assisted death.

\section{Appendix}

Text of the 12 vignettes presented to the members of the public prosecution.

Vignette 1

A 71-year-old patient has cancer with extensive bone metastases. There has been an explicit and persistent request for euthanasia. Life expectancy is estimated to be at most one month. The patient's pain cannot be alleviated with morphine.

If, at the request of the patient, the attending physician performs physician-assisted death, and reports this to the coroner, what would be your recommendation for further action?

\section{Vignette 2}

A 68-year-old patient has cancer with extensive bone metastases. Life expectancy is estimated to be at most one month. There is good pain control with morphine. The patient is in a poor physical condition, suffers from extreme fatigue and is confined to bed. The patient considers the situation to be degrading and persistently makes an explicit request for euthanasia.

If, at the request of the patient, the attending physician performs physician-assisted death, and reports this to the coroner, what would be your recommendation for further action?

Vignette 3

A 70-year-old patient has multiple sclerosis which severely impedes speech; the patient is totally dependent on help with eating, washing, dressing and going to the toilet. The patient is becoming increasingly dizzy and sight is failing. Communication is becoming almost impossible. In spite of a recent relapse in health status, life expectancy is estimated to be at least six months. The patient is said to be suffering unbearably because of his loss़ी of dignity.

If, at the request of the patient, the attending physician performs physician-assisted death, anof reports this to the coroner, what would be youkn recommendation for further action?

Vignette 4

A 71-year-old patient has cancer with bone metastases. The patient is almost constantly suffering from intense pain which - even with $\vec{w}$ morphine - cannot be controlled. There has beens an explicit and persistent request for life-ending Life expectancy is estimated to be at least six months.

If, at the request of the patient, the attending physician performs physician-assisted death, and reports this to the coroner, what would be yourn recommendation for further action?

Vignette 5

A 69-year-old patient has cancer with metastases to bones and brain. For the past few days the patient has no longer been conscious, but is obviţ ously suffering from intense pain which cannot be completely alleviated by morphine. Life expect ancy is estimated to be at most one week. In the past the patient has requested life-ending if the suffering became unbearable.

If, at the request of the partner of the patient $\overline{\bar{p}}$ the attending physician performs physician $\frac{3}{5}$ assisted death, and reports this to the coroner what would be your recommendation for furthes action?

Vignette 6
A 67 -year-old patient is seriously paralysed after several strokes, is no longer able to speak, which? makes communication almost impossible, and is totally dependent on assistance. In the past the patient has told the physician he wants euthanasia if life becomes degrading. The life expectancy is probably more than six months.

If, at the request of the partner of the patient ${ }_{0}^{N}$ the attending physician performs physician $N$ assisted death, and reports this to the coronero what would be your recommendation for furthero action?

Vignette 7

A 73-year-old patient has incipient dementia and cancer with multiple metastases. The patient is confined to bed, is in a poor physical condition, is incontinent for urine and faeces and sometimes asks "to end it all". However, further discussion concerning this request is impossible. Life expecto응 ancy is estimated to be at most one week.

If, at the request of the partner of the patient $\frac{0}{2}$ the attending physician performs physician:- 
assisted death, and reports this to the coroner, what would be your recommendation for further action?

\section{Vignette 8}

A 72-year-old patient has incurable cancer of the pancreas, with severe pain which cannot be alleviated by morphine. During the past two days consciousness has diminished, but the patient is obviously suffering. Life expectancy is estimated to be at most four weeks. In recent weeks the patient has made several requests for an "injection" if nothing else is possible.

If the attending physician performs physicianassisted death, and reports this to the coroner, what would be your recommendation for further action?

\section{Vignette 9}

A 69-year-old cancer patient is rapidly deteriorating, but is still able to communicate. The patient is being fed via a nasogastric tube, and is suffering from increasing pain which cannot be alleviated. Life expectancy is estimated to be a few days. The patient has always refused to talk about the end of life.

If, at the request of the patient's partner, the attending physician performs physician-assisted death, and reports this to the coroner, what would be your recommendation for further action?

\section{Vignette 10}

A 66-year-old patient who has had serious dementia for the past five years, and a slowly progressive form of cancer, is bedridden, very agitated and has extensive bed sores. Life expectancy is estimated to be at most four weeks. Since the patient has been given morphine there have been no more signs of pain. The partner considers the situation to be extremely degrading. No request has been made and there is no living will.

If, at the request of the patient's partner, the attending physician performs physician-assisted death, and reports this to the coroner, what would be your recommendation for further action?

Vignette 11

A 65-year-old patient has cancer with brain metastases. Morphine, administered via a pump, gives good pain control. For the past few days the patient has no longer been conscious, has developed serious bed sores and is increasingly vomiting faeces. Life expectancy is estimated to be at most one week. The partner requests ending of life because the situation is degrading. Neither euthanasia nor physician-assisted suicide have ever been discussed.

If, at the request of the patient's partner, the attending physician performs physician-assisted death, and reports this to the coroner, what would be your recommendation for further action?

\section{Vignette 12}

A 71-year-old patient has cancer of the colon, which cannot be treated. The tumour has grown into the abdominal wall. The patient is severely demented, and conversation is no longer possible. It is evident that there is pain which cannot be alleviated. Life expectancy is estimated to be no more than four weeks. No request has been made and there is no living will.

If, at the request of the partner's patient, the attending physician performs physician-assisted death, and reports this to the coroner, what would be your recommendation for further action?

Facqueline M Cuperus-Bosma, LLM, MD, PhD, is a Researcher in the Department of Social Medicine and the Institute for Research in Extramural Medicine, Vrije Universiteit Amsterdam, Amsterdam, the Netherlands. Gerrit van der Wal, MD, PhD, is Professor of Social Medicine in the same department. Caspar WN Looman, Msc, is a Statistician in the Department of Public Health, Erasmus University, Rotterdam, the Netherlands and Paul $\mathcal{F}$ van der Maas, MD, PhD, is Professor of Public Health in the same department.

\section{References}

1 Wal G van der, Dillmann RJM. Euthanasia in the Netherlands. British Medical fournal 1994;308:1346-9.

2 Wal G van der, Maas PJ van der, Bosma JM et al. Evaluation of the notification procedure for physician-assisted death in the Netherlands. New England fournal of Medicine 1996;335:170611 .

3 Cuperus-Bosma JM, Wal G van der, Maas PJ van den. Physician-assisted death: policy-making by the Assembly of Prosecutors General in the Netherlands. European Fournal of Health Law 1997;4:225-38.

4 Liang, K-Y, Zeger SL. Regression analysis for correlated data. Annual Review of Public Health 1993;14:43-68.

5 In case of univariate comparison (as for type of respondent) it degenerates into testing difference of probability (of a recommendation for an inquest) under assumption of binomial error, but it also offers the possibility of testing differences after correction for other variables (multivariate regression analysis). An introduction can be found in: Dobson AJ. An introduction to statistical modelling. London: Chapman \& Hall, 1983; Jongman RHG, Braak CJF ter, Tongeren OFR van. Data analysis in community and landscape ecology. Cambridge: Cambridge University Press, 1995.

6 Beauchamp TL, Childress JF. Principles of biomedical ethics [4th ed]. New York: Oxford University Press, 1994.

7 Supreme Court 21-6-1994. Nederlandse Jurisprudentie 1994, 656. 